\title{
Gold highlights at the Third International NanoMedicine Conference, in Coogee Beach, Sydney, Australia, 2-4 July 2012
}

\author{
Sónia Carabineiro
}

Published online: 6 November 2012

(C) The Author(s) 2012. This article is published with open access at Springerlink.com

This meeting was attended by 240 participants from diverse fields of nanomedicine. Most presentations were from Australia; however, around 40 delegates were from other countries (including New Zealand, Thailand, Taiwan, China, Hong Kong, India, UK, Hungary, Latvia, Czech Republic, Germany, Italy, Netherlands, France, Portugal and USA). Most attendants were academics but some participants from the industry were also present. Gold-related subjects had an importance presence, showing that gold is very promising for nanomedicine, which aims to treat and prevent disease and traumatic injury, relieving pain and improving human health, using molecular tools and molecular knowledge of the human body (European Science Foundation definition). The topics at the conference ranged from theranostics (a proposed process of personalised medicine consisting on diagnostic therapy for individual patients), drug delivery (administration of a pharmaceutical compound to achieve a therapeutic effect), nanomedicine analytics (techniques used in nanomedicine to detect, analyse, quantify and characterise), macromolecular design (synthesis of large molecules for several nanomedicine applications) and regeneration (process of renewal, restoration and growth to fight disturbance or damage), among others. A brief description is indicated below.

\section{Delivery}

In an oral presentation entitled "Nanoparticles for cancer treatment”, Nicole Bryce (University of Sydney, Australia)

S. Carabineiro $(\square)$

Laboratory of Catalysis and Materials,

Department of Chemical Engineering, Faculty of Engineering,

University of Porto,

Rua Dr. Roberto Frias, s/n,

4200-465 Porto, Portugal

e-mail: scarabin@fe.up.pt showed that sterically stabilised nanoparticles could penetrate spheroid solid tumour models prepared from DLD-1 (colorectal adenocarcinoma) cancer cells. Moreover, the sterically stabilised particles could facilitate the penetration of the chemotherapeutic drug doxorubicin into the spheroid. The speaker demonstrated that the type of steric stabiliser used was the most important, whereas the nature of the solid core particles was relatively unimportant with cores of iron oxide, silica and gold all behaving in a similar manner when similarly stabilised.

Cyrille Boyer (University of New South Wales, Australia) gave an invited oral presentation on "Engineering polymeric nanoparticles for nanomedicine". The speaker showed that soft core-shell polymeric nanoparticles have potential advantages in the sustained and targeted delivery of therapeutic payloads and could offer significant improvements in the temporal and spatial control of drug delivery. The synthesis of new hybrid organic/inorganic nanomaterials, based on iron oxide, gold and gadolinium, was reported for use as contrast agents. These compounds can be used to improve the visibility of internal body structures, after oral or intravenous administration.

Mariana Beija (University of New South Wales, Australia) presented a poster dealing with "Gold nanoparticles as dual contrast agents for magnetic resonance imaging/Xray computed tomography (MRI/CT)". It is important to understand that MRI is a medical imaging technique that uses nuclear magnetic resonance to visualise internal structures of the body in detail and is especially adequate to soft tissues. CT uses computer-processed X-rays to produce tomographic images or 'slices' of specific areas of the body, especially bones and lungs; the cross-sectional images are used for diagnostic and therapeutic purposes in various medical disciplines. If both techniques are combined, a more complete diagnosis can be achieved and the administration of contrast agents into patients with the aim of enhancing the signal-to-background ratio is standard practice, and that is 
what these authors intended. In that scope, they developed macromolecular carriers that improved the relaxivity properties of Gd(III) complexes [1] and prepared gold nanoparticles that were modified with these macromolecular carriers through a 'grafting to' strategy (Fig. 1). These nanohybrids can act as dual contrast agents for MRI and CT and the addition of gold can enhance contrast six times more than when $2,2^{\prime}, 2^{\prime \prime}-(1,4,7,10$-tetraazacyclododecane-1,4,7triyl) triacetate monoamide-Gd(III) complex is used.

The poster of Jane Phui Mun Ng (University of Technology, Sydney, Australia) dealt with "Cellular interactions and cytotoxicity of gold nanoparticles with endothelial cells". It was explained that gold nanoparticles not only provide high contrast in X-ray imaging systems, but are also inert to oxidation, can be modified with fluorescent tags for optical microscopy and can be stabilised against aggregation and modified for increased biocompatibility. Moreover, as gold is non-toxic, the toxicity of gold nanoparticles is dependent on the surface modifying material and size. Once introduced into the human body, they will have contact with the main barriers and lining surfaces, such as the epithelial and endothelial cells, which are commonly employed for the study of cytotoxicity. Therefore, this work focused on antibody CD31 (or platelet endothelial cell adhesion molecule-1, a glycoprotein membrane mediating endothelial cell-cell adhesion involved in angiogenesis for the formation of new vessels). Surface-modified $20 \mathrm{~nm}$ Au nanoparticles (NPs) were prepared through self-assembly of polyethylene glycol (PEG) with thiol(-SH) groups for gold. The resulting PEGmodified nanoparticulate systems exhibited superior colloidal stability and limited toxicity, and binding to non-specific proteins and cells was minimised, enabling their delivery to targeted sites.

\section{Nanomedicine analytics}

Mariam Darestani (University of Sydney, Australia) gave an oral presentation on "Label-free biosensing of protein/antibody interaction". She explained that biosensors are devices designed to detect or quantify biochemicals and that electrical biosensors, including voltammetric, amperometric/coulometric and impedance sensors, are very promising and affordable diagnostic detectors, as they are cheap, low power and easy to miniaturise. Moreover, electrochemical impedance spectroscopy (EIS) technique is a label-free process that does not need any reagent (and impedance is measured as a ratio of current and voltage).

In Darestani's work, protein A (a surface protein found in the cell wall of Staphylococcus aureus) was covalently immobilised on the surface of a self-assembled monolayer on gold and directly on a gold surface. The binding processes were captured by EIS that was able to detect and characterise all the steps of the chemistry and protein/antibody interactions. The direct binding of protein A on gold surface resulted in a capacitance decline at low frequencies, as shown in Fig. 2. This figure also shows that almost $90 \%$ of the binding process occurred in the first $2 \mathrm{~h}$ and the binding process was completed in less than $9 \mathrm{~h}$.

Alice Kar Lai Yang (Chinese University of Hong Kong) gave an oral presentation on "Detection of Methicillinresistant Staphylococcus aureus bacteria (MRSA) deoxyribonucleic acid (DNA) by isothermal amplification (LAMP) with gold nanoparticles by resistive pulse sensing". It was explained that loop-mediated isothermal amplification (LAMP) is a powerful technique to amplify target DNA from pathogens [2] and also a high sensitive and rapid detection method as it can amplify genes within $30 \mathrm{~min}$ at $\sim 60{ }^{\circ} \mathrm{C}$ that can measure complementary DNA or RNA quantitatively.

In Yang's work, Au NPs, acting as carriers or probes for biosensing, were coated with DNA and used for detecting the leukocidin toxic gene $(p v l)$ of MRSA. After LAMP, the $p v l$ amplicons were mixed with the DNA probes on the surface of Au NPs. As a result of the DNA-DNA hybridization, Au NPs formed aggregations. Analysis by resistive pulse sensing provided information on the size and dynamics of the agglometated Au NPs. This sensitive technique could detect up to 100 copies of MRSA templates within a short period of time. The LAMP-based AuNP-RSP showed
Fig. 1 Structure of the dual contrast agent for MRI and CT (adapted from the abstract), consisting in gold nanoparticles modified with $2,2^{\prime}, 2^{\prime \prime}-(1,4,7$, 10 -tetraazacyclododecane-1,4, 7-triyl) triacetate monoamideGd(III) complexes

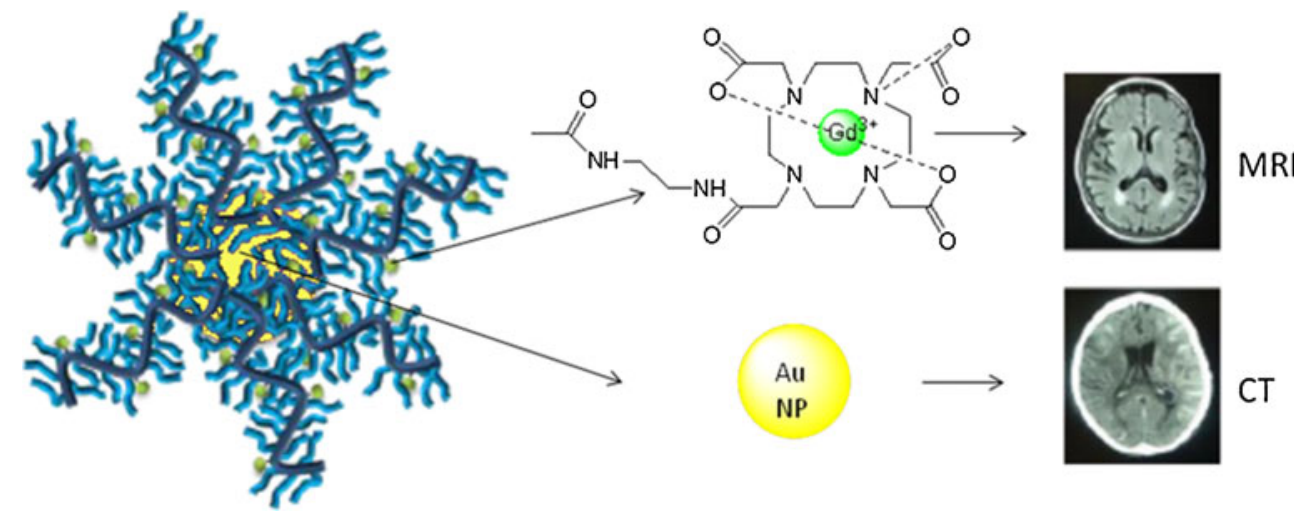


Fig. 2 Capacitance profile of $S$. aureus protein - a binding process in a gold surface (adapted from the abstract)

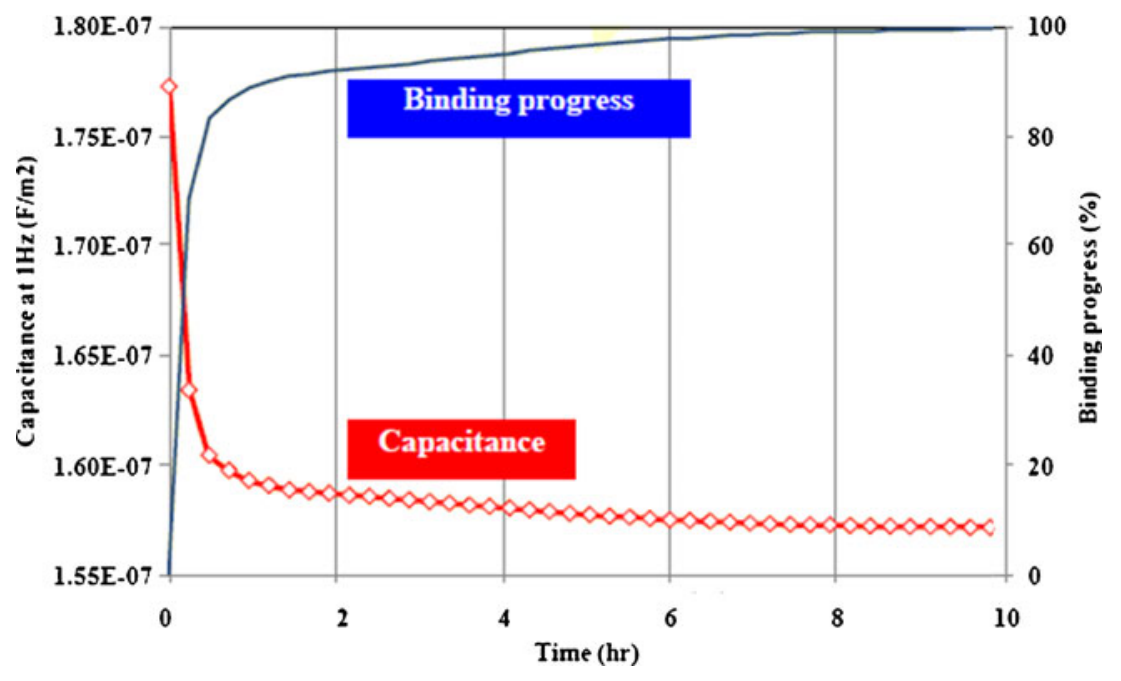

to be a simple and sensitive way to detect different kinds of antigens.

Maitreyee Roy (National Measurement Institute, Australia) gave an oral presentation on "Asymmetric flow-field flow fractionation technique for separation and characterisation of gold nanoparticles in aqueous media". Roy explained that Au NPs are a preferred material for biomedical research into challenging applications, such as advanced diagnostics, drug delivery and therapeutics and that particle size and size distribution are important physical-chemical key characteristics required for describing the nanoparticle systems. Moreover, the asymmetric flow-field flow fractionation (AF-FFF) is a high-resolution elution technique that separates suspension constituents based on hydrodynamic size and a powerful size-based separation method of the components of complex particle suspensions, facilitating determination of chemical composition [3].

In Roy's work, a tri-modal suspension of citratestabilised Au NPs, created by mixing three different sizes (20, 40 and $60 \mathrm{~nm}$ ), at ratios chosen to generate equal volume fractions, was measured with the AF-FFF system. Distinct populations of gold nanoparticles were separated chromatographically from the tri-modal mixture as shown in Fig. 3.

William Olds (Queensland University of Technology, Australia) presented a poster dealing with "Deep Raman spectroscopy for sub-surface probing within biological tissue". Until recently, Raman spectroscopy was considered only a surface analysis technique, but new methods have extended the working depth of Raman spectroscopy to several millimetres, and up to $\sim 1$ to $2 \mathrm{~cm}$, beneath diffuse opaque samples. This explains why Raman is a potential tool for performing non-invasive biomedical spectroscopy and diagnosis.

In Olds's work, the authors constructed a optical probe for conducting Raman spectroscopy through layers of biological media using gold nanoparticles to provide surface- enhanced Raman scattering (SERS), thereby enhancing the signals of buried analytes. In the pilot tests, small samples of chicken skin (both with and without fat layer intact) were used as a model tissue and methylene blue was used as the detection analyte. A strong enhancement of the Raman signals of the methylene blue was observed in the presence of gold nanoparticles. Without the nanoparticles and resultant SERS enhancement, the methylene blue signals could not be detected behind the skin due to the low concentration. Although further work is required to increase the sensitivity of the constructed Raman probe and to optimise the SERS enhancements provided by the gold nanoparticle substrate, these results indicate the possibility of using Raman as a non-invasive biomedical analysis technique, with potential applications in disease diagnosis, blood analyte and drug delivery monitoring.

Chi-Chang Lin (Tunghai University, Taiwan) presented a poster dealing with "Rapid discrimination of bacteria

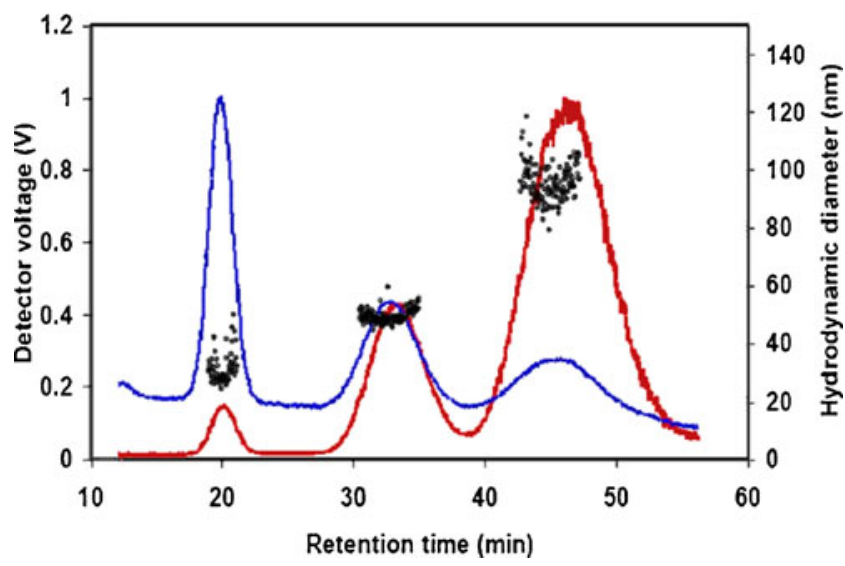

Fig. 3 Fractograms for a tri-modal sample of citrate-stabilised gold nanoparticles. Red line multi-angle static light scattering at $90^{\circ}$; blue line IV-Vis absorption at $520 \mathrm{~nm}$; circles hydrodynamic diameter measured as a function of time by the dynamic light scattering detector (adapted from the abstract) 
fingerprint by using SERS". Lin explained that since infectious diseases are one of the major causes of morbidity and mortality in humans throughout the world, rapid and more accurate methods of screening, identification and susceptibility testing of bacteria are needed.

In Lin's work, a filter-like substrate with high SERS functions made by metal nanoparticles-embedded mesoporous silica was applied for discrimination of bacteria fingerprint under SERS analysis. Gelatine with amide groups $\left(-\mathrm{CO}-\mathrm{NH}_{2}\right)$ was introduced as organic template of mesoporous silica and may also act as the protecting agent for stabilising Au NPs and to prevent their aggregation. The method was simple, low cost, and a strong adsorption affinity to $S$. aureus and Escherichia coli was found. The intensity of $S$. aureus SERS spectrum was enhanced more than 700 times compared with normal Raman spectra upon a $13 \mathrm{wt} \% \mathrm{Au}$ loading.

The poster of Wen-Fu Lai (Taipei Medical University, Taiwan) dealt with "Early detection of prostate cancers and their bone metastasis using near-infrared fluorescent imaging". It was explained that prostate cancer is one of the leading causes of cancers to death in men worldwide, and the metastasis of prostate cancer to bone is the most significant cause of mortality. Therefore, early detection of prostate cancer metastasis to bone is needed for better diagnosis of the disease and available imaging strategies for assessing bony lesions are still lacking.

In Lai's work, a near-infrared fluorescent (NIRF) bisphosphonate derivative probe was designed with ideal optical properties and hydroxyapatite (HA) binding efficacy (HA was selected as a target for a molecular imaging probe because HA deposits at tumour sites of bone metastasis of prostate cancer). Pamidronate (Fig. 4) was selected as the backbone for the molecular probe because of its high affinity towards HA as well as for its small molecular weight. This molecule was conjugated with a nanogold cluster bearing a NIFR fluorescent ligand that can be detected using a in vivo imaging system with adequate filtering. Molecular imaging in animal model was done with NIFR, a technique

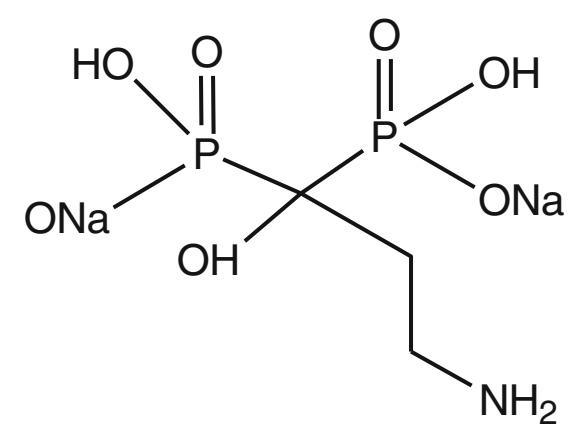

Fig. 4 Structure of pamidronate, a nitrogen-containing bisphosphonate that was conjugated with a nanogold cluster bearing a nearinfrared fluorescent molecular ligand, to be used as a molecular probe that allows a high-resolution image to be taken of the probe due to its ability to penetrate the skin layer and absorb the fluorescence emitted by the probe. The results showed that this probe could specifically and efficiently detect the new bone formation by metastatic prostate cancer in animal models.

Grainne Moran (University of New South Wales, Australia) presented a poster dealing with "Analytical and particle characterisation techniques applied to gold nanoparticles in aqueous media". In a collaboration between the National Measurement Institute Nanometrology Group and the University of New South Wales, techniques for characterising modified Au nanoparticles in aqueous media were investigated. Particle sizes, size distributions and aggregation behaviour were monitored by nanoparticle tracking analysis and differential centrifugal sedimentation, as well as transmission electron microscopy and optical spectroscopy. Cysteine-modified Au NPs with primary particle diameters ranging from 25 to $50 \mathrm{~nm}$ were used as model systems. The information provided by each technique was distinct and complementary. The authors concluded that methods need to be validated using appropriate standards, where available or, at least, crossreferenced using multiple techniques.

\section{General}

Sónia Carabineiro (Universidade do Porto, Portugal) presented a poster dealing with "Uses and applications of gold in nanomedicine". A historical perspective on the use of colloidal gold in medicine throughout the ages was shown, starting from ancient times, where alchemists worked on the development of a gold elixir, which supposedly had the ability to restore health and youth. More recently, gold nanoparticles have been used in the treatment of rheumatoid arthritis due to their anti-inflammatory properties and seem promising for the treatment of Alzheimer's disease. Colloidal methods are often employed for their synthesis (Fig. 5).

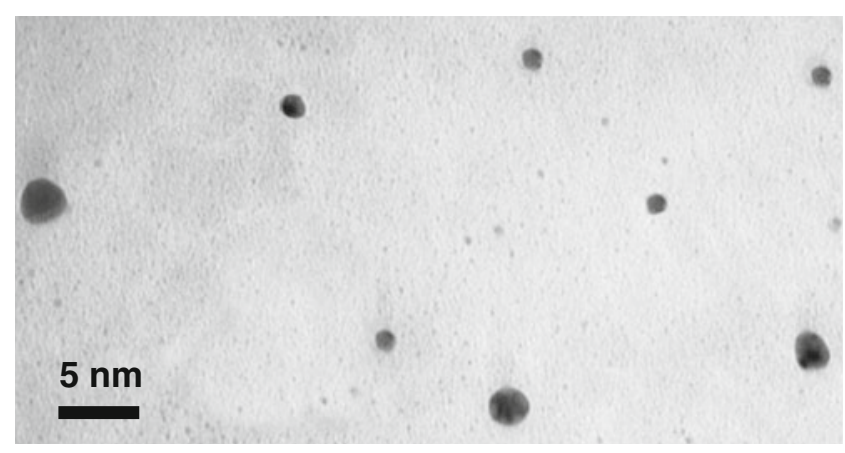

Fig. $5 \mathrm{Au}$ NPs synthesised by a colloidal method using tetrakis (hydroxymethyl) phosphonium chloride to reduce a $\mathrm{HAuCl}_{4}$ solution 
Examples from a recent review [4] were given on the use of gold as a contrast agent in biological electron microscopy or computed tomography; in cancer research, to target tumours and provide detection and also as a drug carrier. Au NPs showed to be effective for the inhibition of pathogenic bacteria cell growth, including Gram-positive bacteria, Gram-negative bacteria and antibiotic-resistant bacteria and have demonstrated effective photothermal destruction of cancer cells and tissue. They can act as antennas, providing enhanced radiation targeting with lower radiation doses, consequently avoiding damage to healthy tissues. This shows that gold has already an important role in nanomedicine. Its potential is enormous and certainly future results will lead to more practical and commercial applications.

Acknowledgments I am grateful to Nicole Bryce and to Carla Gerbo for all the information and assistance provided. Fundação para a Ciência e Tecnologia (FCT) is acknowledged for funding (CIENCIA 2007 programme and project PTDC/QUI-QUI/100682/2008, financed by FCT and FEDER in the context of Programme COMPETE).
Open Access This article is distributed under the terms of the Creative Commons Attribution License which permits any use, distribution, and reproduction in any medium, provided the original author(s) and the source are credited.

\section{References}

1. Li Y, Beija M, Laurent S, van der Elst L, Muller RN, Duong HTT, Lowe AB, Davis TP, Boyer C (2012) Macromolecular ligands for gadolinium MRI contrast agents. Macromolecules 45:4196-4204

2. Tomita N, Mori Y, Kanda H, Notomi T (2008) Loop-mediated isothermal amplification (LAMP) of gene sequences and simple visual detection of products. Nat Protoc 3:877-882

3. Cho TJ, Hackley VA (2003) Fractionation and characterization of gold nanoparticles in aqueous solution: asymmetric-flow field flow fractionation with MALS, DLS, and UV-Vis detection. Anal Bioanal Chem 398:2003-2018

4. SAC Carabineiro (2012) Synthesis of colloidal and supported gold nanoparticles. In: Jarnagin A, Halshauser L (eds) Gold nanoparticles: synthesis, optical properties and applications for cancer treatment, Nova Science, New York (in press) 